\title{
Dependence of the confinement time of an electron plasma on the magnetic field in a quadrupole Penning trap
}

Open Access

\author{
B M Dyavappa, Durgesh Datar, Prakash and Sharath Ananthamurthy* (D)
}

*Correspondence:

asharath@gmail.com

Department of Physics, Bangalore

University, Bangalore, India

\begin{abstract}
A quadrupole Penning trap is used to confine electrons in weak magnetic fields. Perturbations due to space charge and imperfections in the trap geometry, as well as collisions with the background gas molecules, lead to loss of the electrons from the trap. We present in this work the results on measurements of the electron confinement time and its dependence on the magnetic field in a quadrupolar Penning trap. We describe a method to measure the confinement time of an electron cloud under weak magnetic fields $(0.01 \mathrm{~T}-0.1 \mathrm{~T})$. This time is found to scale as $\tau \propto \mathrm{B}^{1.41}$ in variance with the theoretically expected confinement time that scales as $\tau \propto B^{2}$ for trapped electrons that are lost through collisions with the neutrals present in the trap. A measurement of the expansion rate of the electron plasma in the trap through controlled variation of the trap voltage, yields expansion times that depend on the energy of escaping electrons. This is found to vary in our case in the scaling range $\mathrm{B}^{0.32}$ to $B^{0.43}$. Distorting the geometry of the trap, results in a marked change in the confinement time's dependence on the magnetic field. The results indicate that the confinement time of the electron cloud in the trap is limited by both, effects of collisions and perturbations that result in the plasma loss through expansion in the trap.
\end{abstract}

Keywords: Non-neutral plasma, Confinement time, Anharmonicity, Quadrupole penning trap

\section{Introduction}

An essential requirement of ion traps is to confine electrons or ions for longer periods of time under minimal perturbations, in order to carry out studies on them [1-4]. However, the confinement time is finite due to several reasons that include collisions with the surrounding neutrals, anharmonicities in the trap potential induced by deviations from the ideal geometry of the trap, and in situations involving a large number of charged particles, space charge effects in the trap [5]. Inhomogeneity in the external magnetic fields in case of Penning traps is an additional perturber and contributes to loss of ions $[1,6]$. Confinement times of long durations are realized for high precision measurements after incorporating techniques that restrict these perturbations to minimum levels $[2,7,8]$. In a series of pioneering experiments $[9,10]$ electron confinement in cylindrical Penning traps (CPT) have been investigated. According to a confinement theorem [11-14], an azimuthally symmetric trap cannot exert a net torque on the trapped electron

(c) The Author(s). 2017 licensee Springer on behalf of EPJ. This is an Open Access article distributed under the terms of the Creative Commons Attribution License (http://creativecommons.org/licenses/by/4.0), which permits unrestricted use, distribution, and reproduction in any medium, provided the original work is properly credited. 
cloud and the total angular momentum must remain a constant. This limits the radial expansion of the electron cloud. Furthermore, the electrons or ions cannot escape in the axial direction due to the harmonic potential minimum. Notwithstanding the restrictions on losses imposed by the confinement theorem, losses do occur due to the reasons cited earlier.

In measuring the confinement time of an electron plasma in a CPT it is shown that the confinement time departs from the expected $\mathrm{B}^{2}$ dependence at low pressures and is governed by other loss mechanisms apart from elastic binary collisions with background neutrals present $[9,14]$. Induced asymmetries have been applied to investigate plasma confinement time [10] and these lead to a different dependence on the magnetic field. In work examining the background pressure's effect in addition to the applied magnetic field, $B$, measurements of the variation of the radial density of trapped electrons at the centre of the trap $[12,15]$ reveal that the confinement time varies with $B$ and pressure, $\mathrm{P}$, as $\tau \propto \mathrm{B}^{3 / 2} / \mathrm{P}$. In CPTs asymmetries induced by application of patch potentials limit the confinement time and the latter has been studied as a function of plasma column length and applied magnetic field [16]. Stoneking et. al, have used $\mathbf{E} \times \mathbf{B}$ drifts to establish equilibrium in a purely toroidal electron plasma and confinement times of $\sim 3 \mathrm{~s}$ have been obtained [17].

In this work, we carry out investigations of the dependence of the confinement time of an electron plasma on the external magnetic field in a different trap geometry, namely, that of the Quadrupole Penning Trap (QPT). The CPT geometry and electrode structure allows for trapping higher densities of plasma and combination of oppositely charged species, as in anti hydrogen production $[18,19]$. The QPT provides for greater stability when fewer particles are trapped and thus has seen wide applications in tests of QED involving single ions $[1,13,14,20]$. Unlike in the CPT, the QPT has electrodes that are hyperboloids of revolution. When a larger number of ions are trapped in a QPT, such as in our situation with electrons, it is of interest to examine the confinement time's dependence on $\mathrm{B}$ and whether the $\mathrm{B}^{3 / 2}$ dependence as seen in CPTs is reproducible in a QPT. Our measurements yield a confinement time, $\tau \propto B^{1.41}$. This is a departure from the expected scaling $\tau \propto \mathrm{B}^{2}$ obtained taking into account collisional interactions alone with the external neutrals in the trap $[9,10,16]$, and differs from the scaling law as in CPTs. As a complement to these studies, we have also measured the expansion rate of the plasma cloud, through a controlled variation of the trap potential. These measurements point to the influence of collisions as well as plasma diffusion in determining the dependence of $\tau$ on B. We have also carried out measurements with a trap where the internal geometry is altered. This sharply modifies the confinement time's dependence on B suggesting that anharmonicities induced by geometric distortions play a significant role in determining the scaling law.

The paper is organized as follows: After a discussion of the basic theory governing single particle motion, we present a heuristic argument that establishes the dependence of $\tau$ on $\mathrm{B}$ in the presence of collisions with background gases in the trap. We then present a technique based on LabVIEW for measuring the same. This is followed by results from a technique that estimates the plasma expansion time and the dependence on the magnetic field of this expansion. Further, we have carried out measurements of $\tau$ 's dependence on B when the internal geometry of the trap is perturbed. We summarize the results and draw conclusions based on the results. 


\section{Theory}

Penning traps are widely used as devices for confining non-neutral plasmas and fabricated in many geometrical shapes. An abundance of literature exists on Penning traps and their applications for different geometries [21-23]. We briefly summarize the principle of single charged particle confinement in a Penning trap [1]: A quadrupole Penning trap confines charged particles through combining a static electric potential with a uniform magnetic field $\mathrm{B}$ along the symmetry axis of the trap. The quadrupole potential governing single particle motion is described by

$$
\Phi(x, y, z)=\frac{U_{0}}{r_{0}^{2}+2 z_{0}^{2}}\left(2 z^{2}-x^{2}-y^{2}\right)
$$

Where $U_{0}$ is the applied potential; $r$ and $z$ are the radial and axial coordinates respectively; and $d$ is the characteristic dimension of the trap given by $d^{2}=r_{0}^{2}+2 z_{0}^{2}, r_{0}$ being the radius of the ring electrode and $z_{0}$, is half the distance between the two end cap electrodes. Figure 1 shows the electric and magnetic field lines in the trap. The degrees of freedom in the $x$ and $y$ directions (radial motion) are coupled, resulting in coupled equations of motion of the electron. On the other hand, motion of the electron along the $z$-direction (axial motion) is uncoupled. The solutions of the equations are obtained by a frame transformation yielding the characteristic frequencies $\left(\omega_{z}, \omega_{c}^{\prime}, \omega_{m}\right)$ of motion for a single ion or electron $[1,2]$, where $\omega_{z}$ is the axial oscillation frequency of motion due to the harmonic potential, $\omega_{c}^{\prime}$ is the modified cyclotron frequency. $\omega_{m}$ is the magnetron frequency representing a drift in the radial plane around the centre of the trap. Note that the axial oscillation has no dependence on the magnetic field and for stable solutions we require that $\omega_{c} \geq \sqrt{2} \omega_{z}, \omega_{c}$ being the cyclotron frequency. In a QPT collisions with the background neutrals lead to instabilities and an expansion in the radial plane (Fig. 2). In a real trap with a cloud of electrons the radial and axial motion of the electrons are coupled due to Coulomb interactions as well as perturbations in the trap potential that originate from imperfections in the trap geometry. This leads to the presence of higher order frequencies measured as the motional resonances, in addition to the single particle frequencies described here $[1,2,24,25]$. The trapped electrons with temperature corresponding to a few $\mathrm{eV}$, constitutes a non-neutral weakly coupled plasma with Coulomb coupling parameter $\sim 10^{-4}$, number density $\sim 10^{+15} \mathrm{~m}^{-3}$, Debye length $\sim 0.1 \mathrm{~mm}$ and plasma cloud of radius $\sim 4 \mathrm{~mm}$. Thus, an independent particle model can be employed in our trapped electron plasma conditions for analysis.

Since the kinetic energy of trapped electrons is a few $\mathrm{eV}$ and comparable to the typical trap depths which is also a few eV, this leads to a high rate of loss of electrons [26]. The hotter electrons collide more frequently with the background molecules of the gas in the trap and consequently, are lost more easily than the colder electrons. Theoretical calculations based on transport theories [27] in the case of Malmberg Penning traps reveal that collisions cause plasma expansion and the rate is proportional to $B^{-2}$. We present here a simple argument to illustrate the dependence of the confinement time on the applied magnetic field. The linear frequency of cyclotron motion may be written as

$$
f_{c}=\frac{q B}{2 \pi m}
$$

$$
f_{c} \propto B
$$




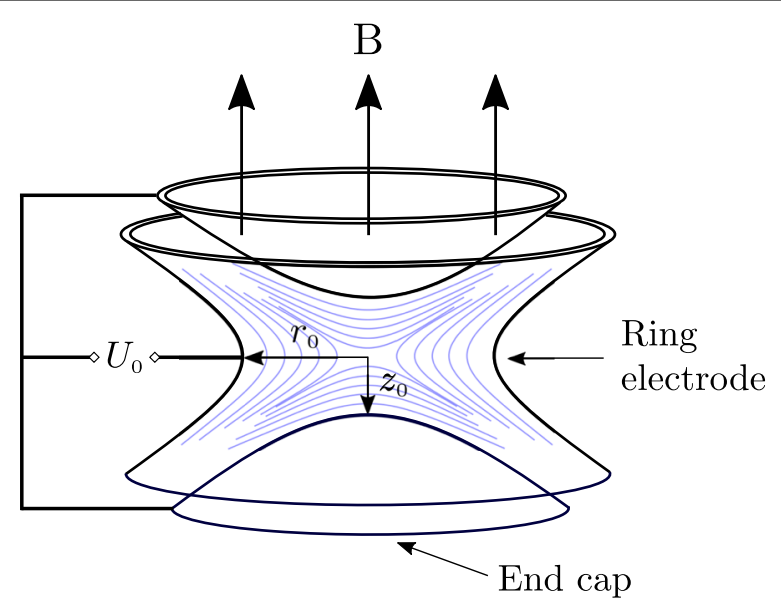

Fig. 1 Quadrupole penning trap, where $r_{0}=7 \mathrm{~mm}$ and $z_{0}=5 \mathrm{~mm}$

The expression for the angular frequency of magnetron motion is given by

$$
\omega_{m}=\frac{\omega_{c}}{2}-\sqrt{\frac{\omega_{c}^{2}}{4}-\frac{\omega_{z}^{2}}{2}}
$$

Since $\omega_{z} \ll \omega_{c}$ we can write up to first order,

$$
\omega_{m} \approx \frac{\omega_{z}^{2}}{2 \omega_{c}}
$$

Therefore the linear frequency of magnetron motion

$$
f_{m} \propto \frac{1}{B}
$$

Increasing the magnetic field thus, increases the cyclotron frequency and decreases the magnetron frequency. As the magnetic field increases, the radius of the cyclotron orbit decreases due the conservation of angular momentum. Therefore,

$$
B \propto \frac{1}{r_{c}}
$$

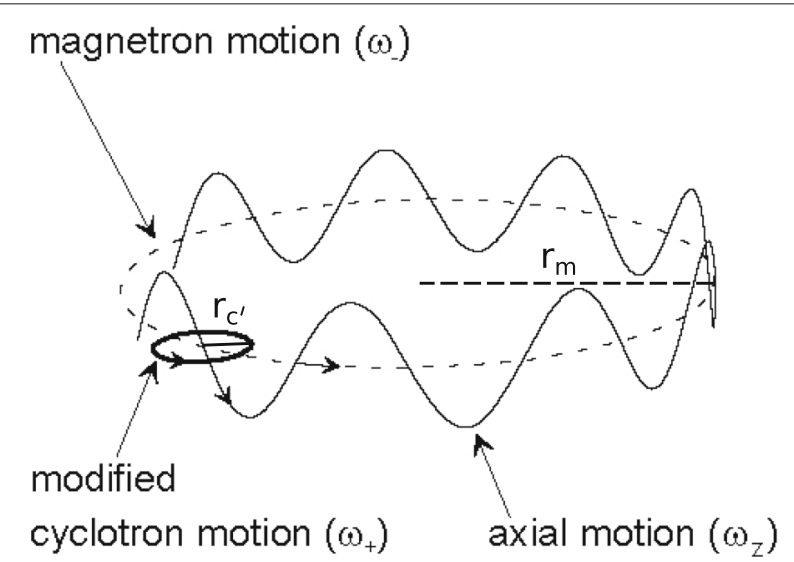

Fig. 2 Single particle motion in QPT. Collisions lead to radial expansion of the particle. $r_{m} \& r_{c}^{\prime} \approx r_{c}$ are respectively the radii of the magnetron and cyclotron motion 
The frequency of collisions, $f$, of a single electron in a cycle of the magnetron motion,

$$
f \propto r_{c^{\prime}} r_{m}
$$

where $r_{c^{\prime}}$ and $r_{m}$ are respectively, the radii corresponding to the (modified) cyclotron and magnetron motions (Fig. 2).

The energy associated with the single particle motion is given by $[28,29]$

$$
H=\frac{1}{2} m \omega_{1} \omega_{c^{\prime}} r_{c^{\prime}}^{2}-\frac{1}{2} m \omega_{1} \omega_{m} r_{m}^{2}+\frac{1}{2} m \omega_{1} \omega_{z}^{2} r_{z}^{2}
$$

where $\omega_{1}=\sqrt{\omega_{c}^{2}-2 \omega_{z}^{2}}$. Note that the contribution to the energy of the particle due to the magnetron motion is negative, implying that collisions will increase $r_{m}$ resulting in particle loss [30, 31]. In order to reduce the second term in Eq.9, $\omega_{m}$ has to be decreased (Eq.6), by increasing B.

Thus,

$$
f \propto \frac{1}{f_{c}} \times f_{m}
$$

or,

$$
\tau \propto \frac{1}{f} \propto \frac{f_{c}}{f_{m}} \propto B^{2}
$$

Thus, collisions alone should limit the confinement time in a manner that determines the variation of the confinement time as illustrated. However as mentioned earlier, a cloud of electron plasma is subject to loss due to expansion of the cloud on account of trap conditions that cause instabilities [9-16]. The total canonical angular momentum must be conserved, therefore the like-particle interactions which are complex and non-linear cannot expand the plasma radially. Torques may arise from collisions with neutral atoms, angular asymmetries in magnetic field or the containment vessel may act to change the total canonical angular momentum and expand the plasma radially.

\section{Experimental details}

Measurement of confinement time

Detection of the trapped electrons is through an electronic tank circuit weakly coupled to the trap. The details of this technique may be found in previous work [1, 24, 25, 32]. The electrons generated from a thoriated tungsten filament are loaded into the trap and the filament current is then switched off while simultaneously setting the storage voltage, $U_{0}$, that is applied between the end cap and ring electrodes. After a fixed duration of 10 ms, starting from the moment the loading of electrons stops, $U_{0}$ is ramped down (Fig. 3a and c). This duration is chosen to be greater than the time for electrons to achieve equilibrium $(10 \mu \mathrm{s})$ [33]. At the voltage value where the corresponding axial frequency coincides with the tank circuit frequency, resonant energy transfer from the circuit to the oscillating electrons results in a dip in the demodulated DC output of the circuit. The extent of the dip is proportional to the number of electrons axially oscillating, for weak excitation of the circuit oscillation. This sequence is then repeated for different time steps. We thus obtain the loss curve of the trapped electrons as a function of time. However the filament current fluctuates, and hence identical loading of electrons into trap in each trial is not assured, apart from the situation here where each timing and detection cycle results in only a single data point in this case. In a different approach (Fig. 3c), the ramp cycle 


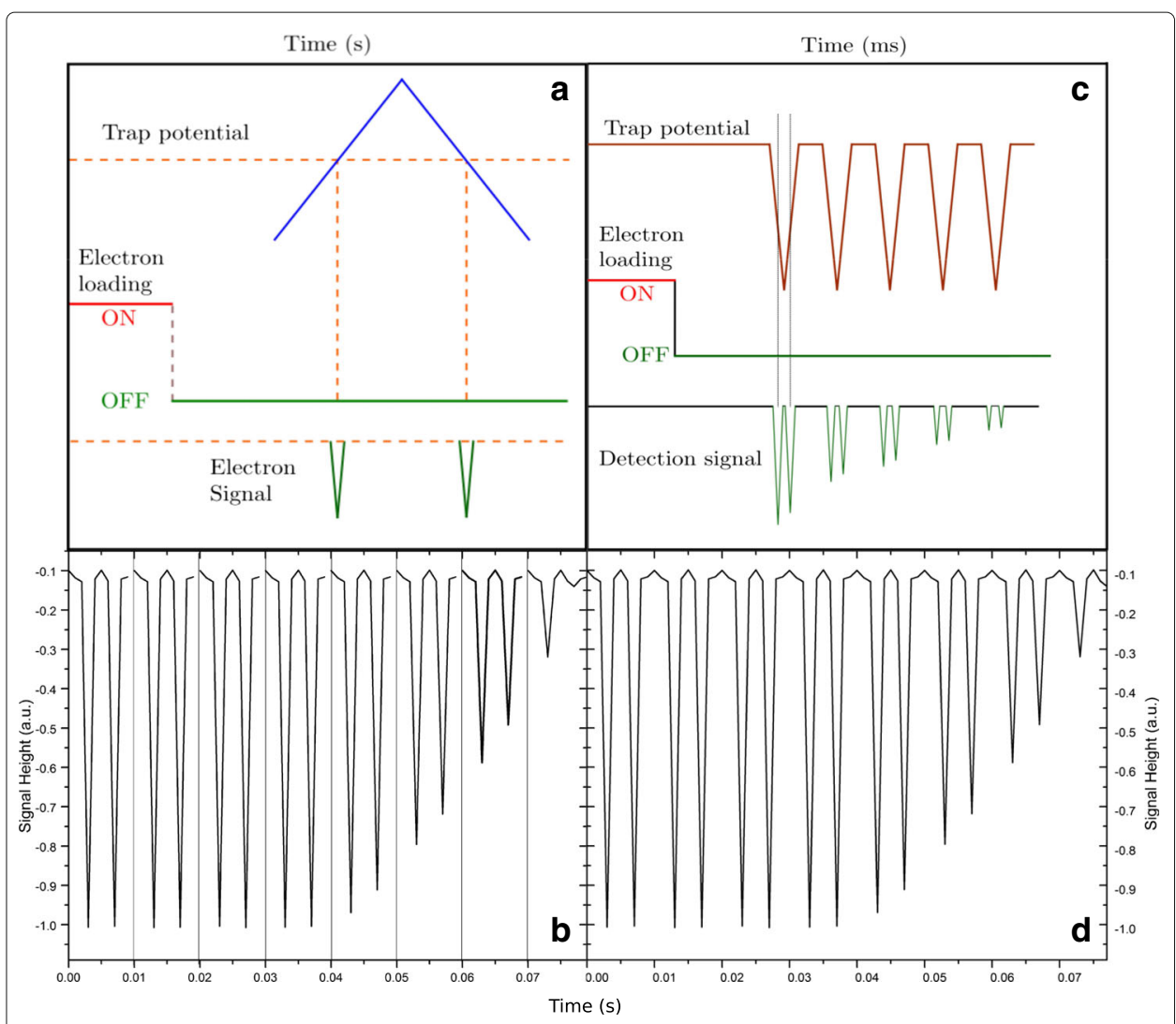

Fig. 3 (a) Schematic diagram of single ramp scanning mode (b) Electron signal fall-off with single ramp yields $\tau=72.89 \mathrm{~ms}$ at a magnetic field of $0.05 \mathrm{~T}$ (c) Schematic diagram of continuous ramps scanning mode (d) Electron signal fall-off with time for continuous ramping yields $\tau=72.32 \mathrm{~ms}$ at a magnetic field of $0.05 \mathrm{~T}$

is continuously repeated at the same ramp frequency soon after loading of electrons is stopped. This results in the collection of a larger set of data points for a single loading event and excludes any variations induced by possible differences in the electron loading. The confinement time of trapped electrons is measured by acquiring the resonance absorption signal. The resonance absorption signal of the trapped electrons was acquired at fixed intervals of time.

The trapped electrons are continuously sampled over time steps of $10 \mathrm{~ms}$. The disadvantage in this manner of probing for stored electrons is in the repetitive detection cycles that may result in perturbative losses due to the excitation from the coupled tank circuit, weak as it may be, overriding the intrinsic loss mechanisms. However, a comparison of data obtained through both methods yielded no significant differences in measurement of the signal fall off features as shown in Fig. 3b and d. Hence, the latter method is adopted as it yields more data points for a single loading detection event. The area under the signal is plotted against the time interval. The measurement of confinement time by the single ramp as well as continuous ramping yield almost the same values, as shown in Fig. $3 b$ and $d$.

The magnetic field at the position of the trap between the poles of the electromagnet was obtained through a measurement of the cyclotron frequency of the electrons in the 
trap by excitation of the motional spectrum through external RF excitation. The procedure for obtaining the motional spectrum is described elsewhere and we will not elaborate this here $[24,25,32]$. The magnetic field obtained by measurement of the cyclotron frequency is plotted against the current in the electromagnet for a few measurements. This yields the magnetic field at the trap center for all currents (Fig. 4). The dimensions of the trap extend $7 \mathrm{~mm}$ radially and $5 \mathrm{~mm}$ axially from the trap center. The magnetic field is measured using a hall probe along the $X, Y$ and $Z$ axes from the centre of the pole pieces of the electromagnet. The magnetic field can be considered to be homogeneous within the dimensions of the trap, within a small variation. Figure 5 shows the magnetic field's variation in space around the center of the pole pieces of electromagnet. The variation in any axis was found to be less than $0.6 \%$. The background pressure in the trap was $2 \times 10^{-8}$ torr, for all measurements.

\section{Measurement of expansion rate of the electron plasma}

The trapped non neutral plasma undergoes losses both through collisions and torques induced on the plasma cloud due to anharmonicities present in the trap. As the confinement time is limited by both, collisions with background neutrals, as well as by the inherent instability of the electron plasma, we measure the expansion rate of the plasma as a function of the magnetic field as described in the following section [12, 15, 27, 34]:

The trap voltage is set at a higher value such as $10 \mathrm{~V}$ to ensure that the entire energy spectrum of electrons is confined by the potential. Loading of the electrons is stopped

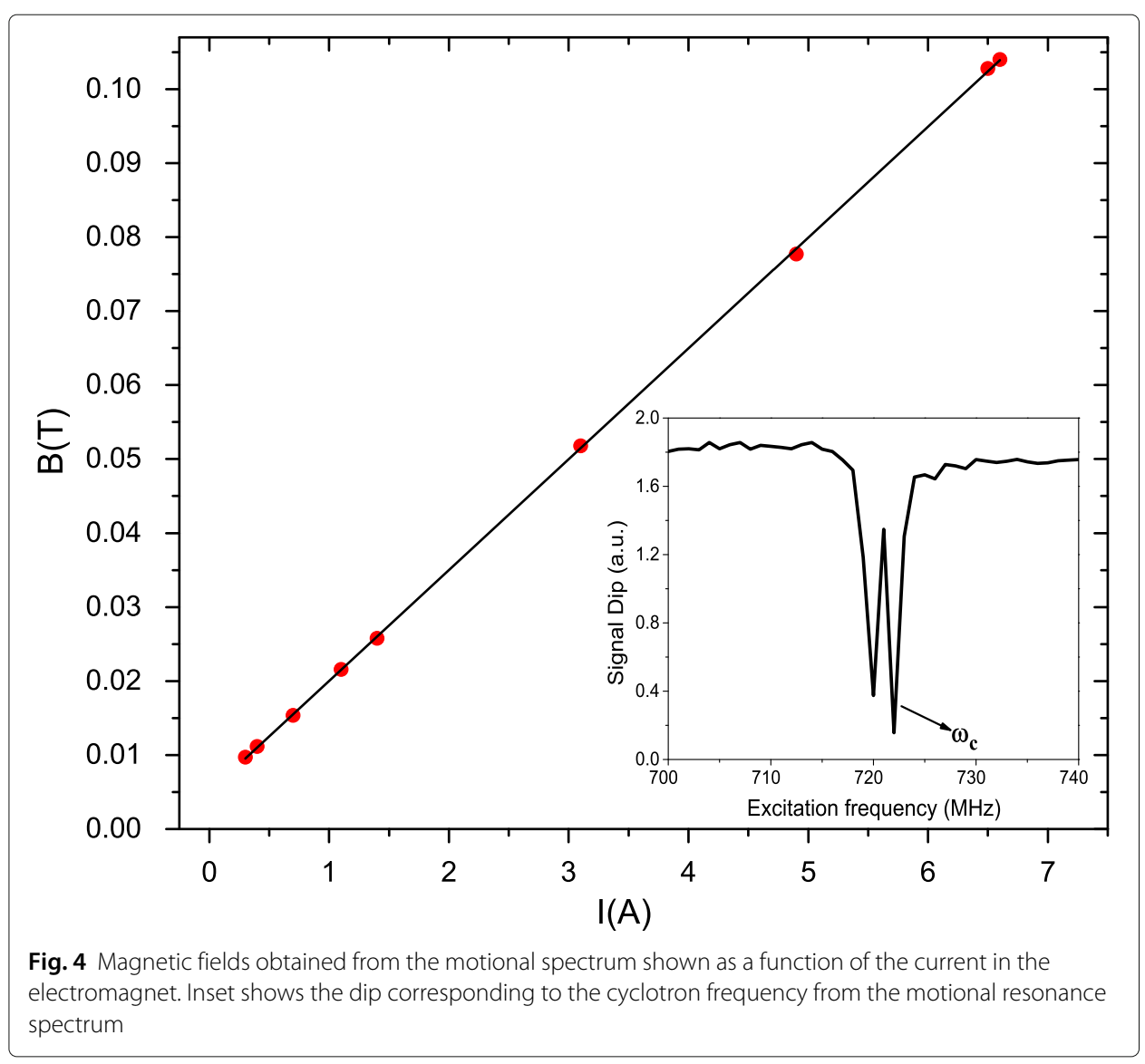






Fig. 5 Homogeneity of magnetic field along $X$, Yand $Z$ axes

and simultaneously the storage voltage is reduced to zero. This results in the escape of electrons, with electrons energies greater than $\mathrm{eV}$ exiting the trapping region faster than the lower energy (less than $\mathrm{eV}$ ) electrons that are confined within the potential. After a certain time, the voltage is ramped up rapidly to a certain voltage $V^{\prime}$. This results in recapture of the electrons that are still in the trapping region with energy range around $V^{\prime}$. The schematic of this sequence is shown in Fig. 6 and shows results of the dwell time measurements before stepping up $V^{\prime}$ to different levels. The time increases as the step up voltage, as expected, since higher step up voltages, results in the recapture of a wider energy range of electrons, whereas lower step up voltages result in capturing only the electrons of energies less than the range corresponding to the lower voltage.

As the axial energy distribution measurements we have carried out indicate, the electron energies are centered around $5 \mathrm{~V}$, the bias on the electron source [33]. This measurement also points to the dependence of the electron cloud expansion rate on the energy range of the electrons, i.e. diffusion of lower energy electrons from the centre of trap is slower than their higher energy counterparts.

The experiment was performed at different magnetic fields and for different times, until the signal cannot be detected at any step up voltage, $V^{\prime}$. The signal decay versus time for different magnetic fields is shown in the Fig. 7 and shows the expansion rate for different magnetic fields for step up voltages of $2 \mathrm{~V}, 4 \mathrm{~V}$ and $5 \mathrm{~V}$. One can note that in all situations measuring the electron loss as a function of time, the signal is initially constant and then sharply drops. The absence of an exponential fall off pattern throughout the measurement time, we conjecture, has to do with the electrons gaining velocity and hence collision frequency as they diffuse out of the trap. The trapped non neutral plasma undergoes losses both through collisions and torques induced on the plasma cloud due to anharmonicities present in the trap. The latter results in the expansion of the plasma [12, 15, 26, 27]. 




Fig. 6 Schematic diagrams showing electron signal along with variation of storage potential in different steps where, $T_{V^{\prime}=0}$ is the time for which the storage potential is zero

\section{Results and discussion}

\section{Dependence on magnetic field}

Figure $3 \mathrm{~b}$ shows the decay of signal area with dwell time recorded in the single ramp mode. All data was recorded at the lowest pressure attainable under normal operating conditions of our setup (low $10^{-8}$ Torr). Figure 8 shows the dependence of B on $\tau$ revealing that $\tau \propto \mathrm{B}^{1.41 \pm 0.04}$.

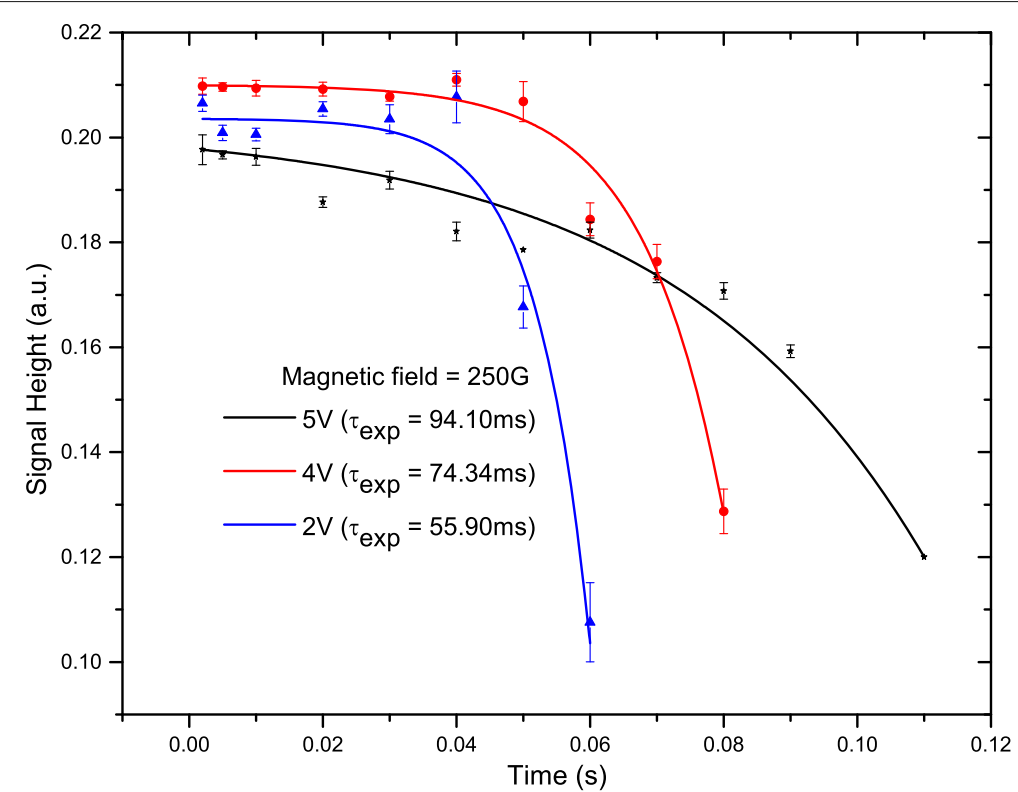

Fig. 7 Electron signal fall off with scanning time at step up voltages of $5 \mathrm{~V}, 4 \mathrm{~V}$ and $2 \mathrm{~V}$ yielding different confinement times 


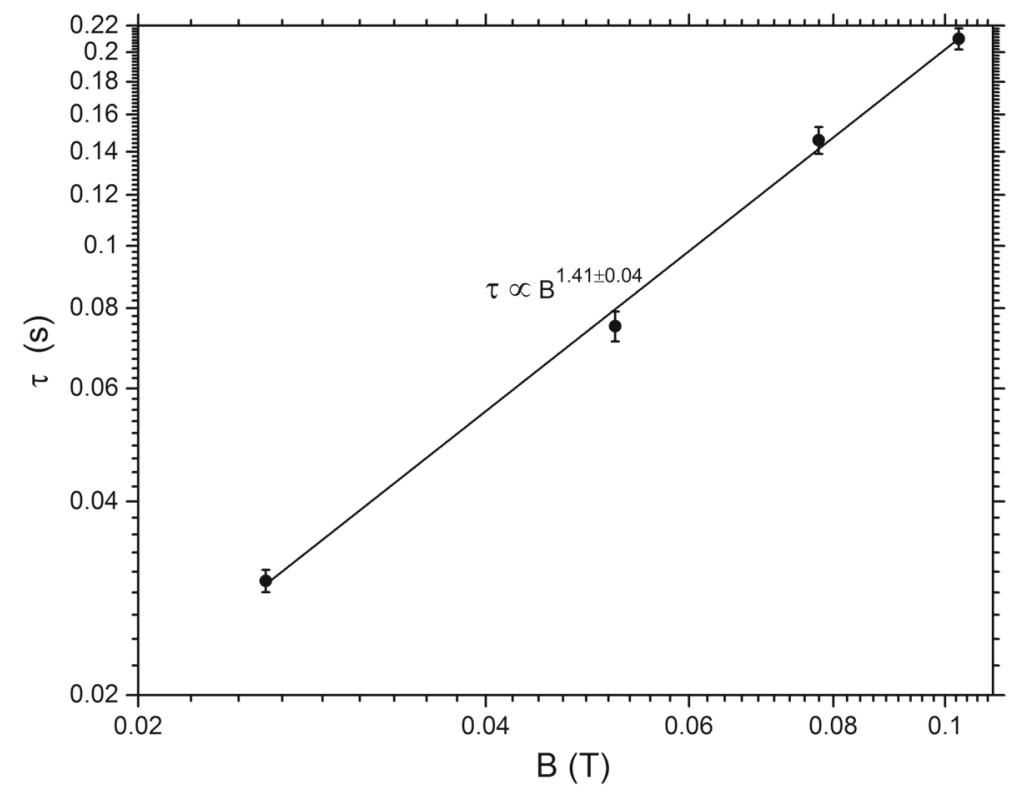

Fig. 8 Confinement time dependence on magnetic field showing $\tau \propto B^{1.41 \pm 0.04}$

\section{Plasma expansion rate dependence on magnetic field}

When the storage potential is made zero, the plasma expands and is lost from the trap in the axial direction primarily, although the nonlinear couplings in the degrees of freedom result in radial expansion as well. The coupling between the radial and axial degrees of freedom leads to a dependence on the magnetic field of the plasma expanding primarily in the axial direction, in this set of experiments.

The slope of the plot of B $-\tau$ on a logarithmic scale yield slopes of $0.32 \pm 0.02$ and $0.43 \pm 0.04$, for step up voltages of $2 \mathrm{~V}$ and $4 \mathrm{~V}$ respectively, as shown in Fig. 9. Therefore $\mathrm{B}$ $-\tau$ graphs show that the plasma expansion time depends on magnetic field in the range $\mathrm{B}^{0.32 \pm 0.02}-\mathrm{B}^{0.43 \pm 0.04}$, indicating the dependence of this expansion on the kinetic energy range of the electrons.

For a strongly magnetized plasma $\omega_{c e}^{2} \gg \omega_{p e}^{2}$, where $\omega_{c e}$ and $\omega_{p e}$ are the cyclotron frequency and the plasma frequency of electron plasma respectively [11] and the plasma expands on a time scale given by the diffusion time, $\tau_{\text {diff }} \sim\left(\omega_{p e}^{2} v_{e n} / \omega_{c e}^{2}\right)^{-1}[1,12,32]$. For an electron density of $10^{15} \mathrm{~m}^{-3}$ and magnetic field of $0.05 \mathrm{~T}$, we obtain the electronneutral collision frequency $v_{e n} \sim 100 \mathrm{~s}^{-1}$ and $\tau_{\text {diff }} \sim 65 \mathrm{~ms}$, which is of the order of the expansion times that we measure.

\section{Induced geometric distortions and confinement time}

A distortion in the trap geometry is induced by pushing the electron filament tip $1 \mathrm{~mm}$ into the volume of the trap, so that it protrudes in as shown in Fig. 10. The confinement time is measured as before for this condition inside the trap:

Figure 11 shows a much reduced power dependence on the magnetic field than in the case without the filament tip protruding into the trap. This points to the sensitive dependence of the confinement time on the geometry of the trap. Further distortion of the trap geometry by pushing the filament tip a little more resulted in no confinement for any magnetic field. 


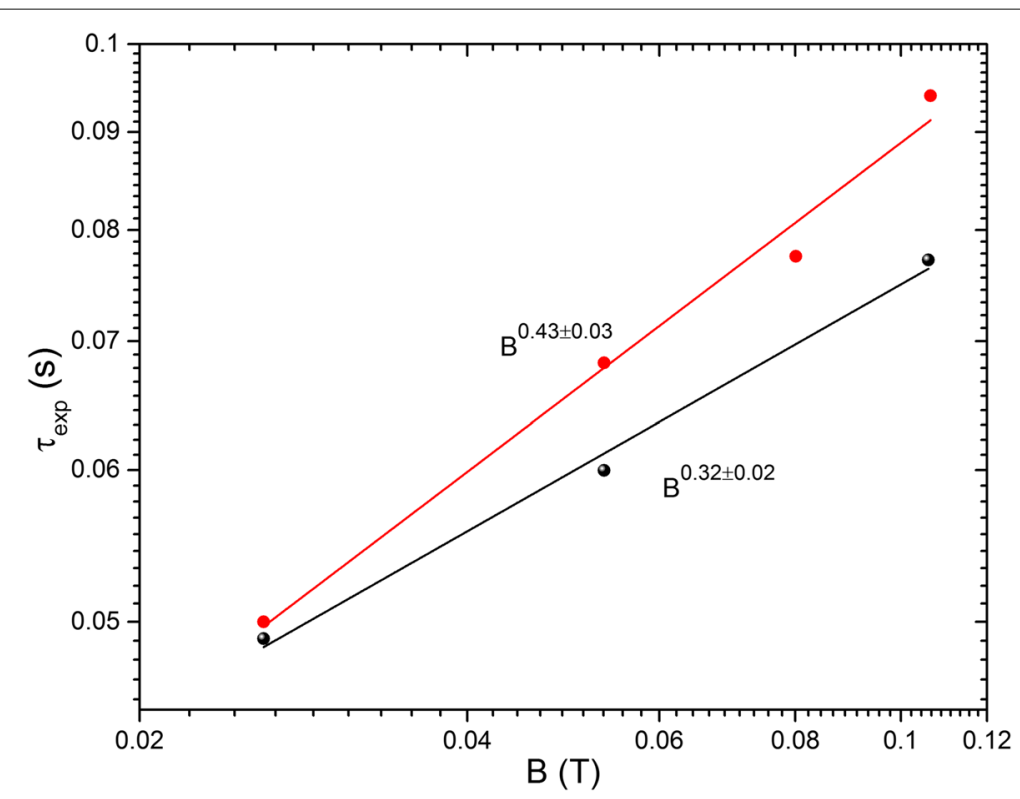

Fig. 9 Expansion time dependence on magnetic field showing the plasma expansion rate (red) for step up of $4 \mathrm{~V}$ and (black) for step up of $2 \mathrm{~V}$

\section{Conclusion}

We have presented results on measurements of the confinement time of an electron plasma in a quadrupole Penning trap, and the dependence of the same on external magnetic fields. The confinement time departs from the $\mathrm{B}^{2}$ dependence that is expected for a few particles confined in harmonic traps. Here, an electron plasma of a few $\mathrm{eV}$ energy and density of $10^{15} \mathrm{~m}^{-3}$ is trapped and this situation enhances space charge effects on

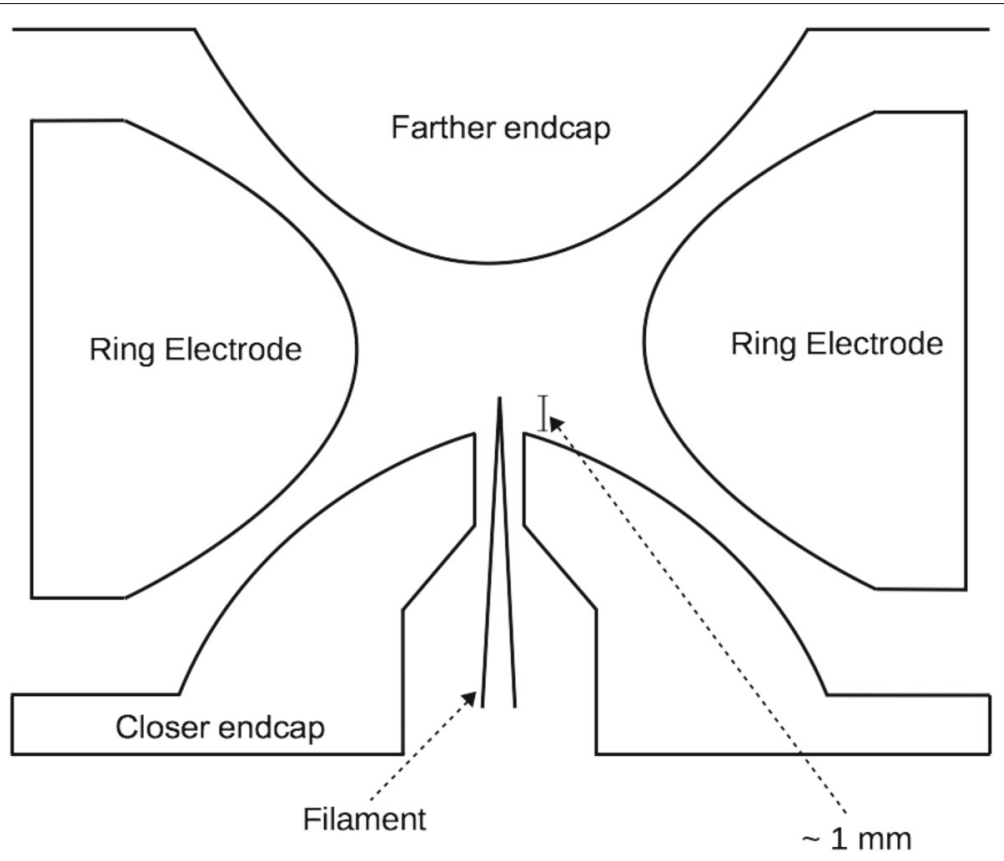

Fig. 10 End cap of trap fitted with protruding filament in the trap volume 


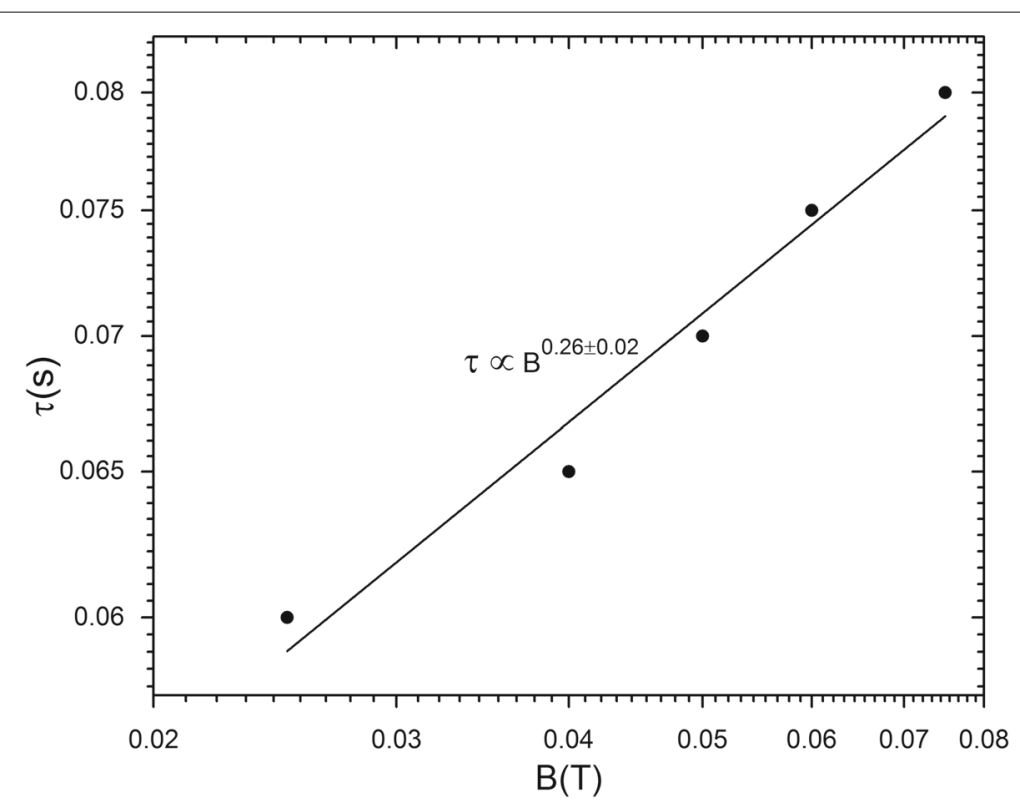

Fig. 11 Confinement time dependence on magnetic field with induced geometric distortions

the individual electron's degrees of freedom [5, 33, 35]. For a quadrupole Penning trap, we report a $\tau$ dependence $\propto B^{1.41 \pm 0.04}$. Controlled expansion of the plasma through varying the trap potential results in axial expansion of the plasma. The magnetic field dependence on this has been studied as well, and this depends on the kinetic energy range of the confined electrons. We have demonstrated through perturbing the internal geometry of the trap that the confinement time's dependence on the magnetic field reduces significantly and was measured to be $\tau \propto \mathrm{B}^{0.26 \pm 0.02}$. Further disturbance of the trap geometry through pushing the filament tip further into the trap resulted in no confinement of the electrons within the magnetic field range used in our experiments, leading us to conclude that the scaling law seems to depend on the nature and extent of the perturbations. Further work to build a theoretical model incorporating higher order perturbative terms in the potential in order to predict the confinement time scaling with $\mathrm{B}$, would help in designing traps that can minimize these perturbative terms $[36,37]$.

Acknowledgements

Prof. G. Werth, University of Mainz, Germany, for useful discussions and suggestions. Dr. Satyajith, K.T. Amrita School of Engineering, Amrita University, for useful discussions and suggestions. DD acknowledges a fellowship from the University Grants Commission, India. SA acknowledges a previous DAE-BRNS grant (2001-2005) that enabled the present experimental work.

Authors' contributions

BMD carried out the measurements and wrote the paper. DD helped develop the codes and programmes in LabVIEW. P assisted in the measurements and the data analysis and SA supervised the measurements and wrote the final version of the paper. All authors read and approved the final manuscript.

Competing interests

The authors declare that they have no competing interests.

\section{Publisher's Note}

Springer Nature remains neutral with regard to jurisdictional claims in published maps and institutional affiliations. 


\section{References}

1. Major FG, Gheorghe VN, Werth G (2005) Charged Particle Traps, vol 37. Springer, Verlag Berlin Heidelberg

2. Ghosh PK (1995) Ion Traps. Clarendon Press, Oxford

3. Dehmelt H (1990) Experiments with an isolated subatomic particle at rest. Rev Mod Phys 62:525-530

4. Paul W (1990) Electromagnetic traps for charged and neutral particles. Rev Mod Phys 62:531-540

5. Winters DFA, Vogel M, Segal DM, Thompson RC (2006) Electronic detection of charged particle effects in a Penning trap. J Phys B Atomic Mol Opt Phys 39:3131

6. Bollen G, Moore RB, Savard G, Stolzenberg H (1990) The accuracy of heavy-ion mass measurements using time of flight-ion cyclotron resonance in a Penning trap. J Appl Phys 68:4355-4374

7. Hall DS, Gabrielse G (1996) Electron Cooling of Protons in a Nested Penning trap. Phys Rev Lett 77:1962-1965

8. Stoneking MR, Growdon MA, Milne ML, Peterson RT (2004) Poloidal $\boldsymbol{E} \times \boldsymbol{B}$ Drift Used as an Effective Rotational Transform to Achieve Long Confinement Times in a Toroidal Electron Plasma. Phys Rev Lett 92:095003

9. Malmberg JH, Driscoll CF (1980) Long-time containment of a Pure Electron Plasma. Phys Rev Lett 44:654-657

10. Driscoll CF, Malmberg JH (1983) Length-Dependent Containment of a Pure Electron-Plasma Column. Phys Rev Lett 50:167-170

11. O'Neil TM (1980) A confinement theorem for nonneutral plasmas. Phys Fluids 23:2216-2218

12. Davidson RC, Moore DA (1996) Expansion and nonlinear relaxation of a strongly magnetized non-neutral electron plasma due to elastic collisions with background neutral gas. Phys Plasmas 3:218-225

13. Dubin DHE, O'Neil TM (1999) Trapped nonneutral plasmas, liquids, and crystals (the thermal equilibrium states). Rev Mod Phys 71:87-172

14. O'Neil TM (1995) Plasmas with a single sign of charge (an overview). Phys Scr 1995:341

15. Davidson RC, Chao EH (1996) Nonlinear expansion and heating of a nonneutral electron plasma due to elastic collisions with background neutral gas. Phys Plasmas 3:2615-2619

16. Notte J, Fajans J (1994) The effect of asymmetries on non-neutral plasma confinement time. Phys Plasmas 1:1123-1127

17. Marler JP, Stoneking MR (2008) Confinement time exceeding one second for a toroidal electron plasma. Phys Rev Lett 100(15):155001

18. Amoretti M, et al (2002) Production and detection of cold antihydrogen atoms. Nature 419(6906):456-459

19. Gabrielse G, Bowden NS, Oxley P, Speck A, Storry CH, Tan JN, Wessels M, Grzonka D, Oelert W, Schepers G, Sefzick T, Walz J, Pittner H, Hänsch TW, Hessels EA (2002) Background-free observation of cold antihydrogen with field-ionization analysis of its states. Phys Rev Lett 89(21):213401

20. Blaum K, Novikov YN, Werth G (2010) Penning traps as a versatile tool for precise experiments in fundamental physics. Contemp Phys 51(2):149-175

21. Gabrielse G (2006) Antiproton mass measurements. Int J Mass Spectrom 251:273-280

22. Stahl S, Galve F, Alonso J, Djekic S, Quint W, Valenzuela T, Verdú J, Vogel M, Werth G (2005) A planar Penning trap. Eur Phys J D Atomic Mol Opt Plasma Phys 32:139-146

23. CASTREJÓN-PITA JR, Ohadi H, Crick DR, Winters DFA, Segal DM, Thompson RC (2007) Novel designs for Penning ion traps. J Mod Opt 54:1581-1594

24. Paasche P, Valenzuela T, Biswas D, Angelescu C, Werth G (2002) Individual and center-of-mass resonances in the motional spectrum of an electron cloud in a penning trap. Eur Phys J D Atom Mol Opt Plasma Phys 18:295-300

25. Paasche P, Angelescu C, Ananthamurthy S, Biswas D, Valenzuela T, Werth G (2003) Instabilities of an electron cloud in a Penning trap. Eur Phys J D Atom Mol Opt Plasma Phys 22:183-188

26. Dinklage A (ed) (2005) Plasma Physics: Confinement, Transport and Collective Effects. Springer, Verlag Berlin Heidelberg. Lecture notes series in Physics

27. Chao EH, Davidson RC, Paul SF (1999) Non-neutral plasma expansion induced by electron-neutral collisions in a Malmberg-Penning trap. J Vac Sci Technol A Vacuum Surf Films 17:2050-2055

28. Horvath GZK, Thompson RC, Knight PL (1997) Fundamental physics with trapped ions. Contemp Phys 38(1):25-48

29. Itano WM, Wineland DJ (1982) Laser cooling of ions stored in harmonic and penning traps. Phys Rev A 25(1):35-54

30. Savard G, Becker S, Bollen G, Kluge HJ, Moore RB, Otto T, Schweikhard L, Stolzenberg H, Wiess U (1991) A new cooling technique for heavy ions in a penning trap. Phys Lett A 158(5):247-252

31. Brown LS, Gabrielse G (1986) Geonium theory: Physics of a single electron or ion in a penning trap. Rev Mod Phys. 58(1):233-311

32. Satyajit KT, Gupta A, Joshi G, Mohan S, Rao P, Ananthamurthy S (2009) Loading Detection and Number Estimation of an Electron Plasma in a Penning Prap. Plasma Sci Technol 11:521

33. Datar D, Dyavappa BM, Mahesh BL, Satyajith KT, Ananthamurthy S (2016) Energy distribution of electrons under axial motion in a quadrupole Penning trap. Can J Phys 94:1245-1249

34. Chang Y, Correa JR, Ordonez CA (2004) Loss rate of an electrically trapped non-neutral plasma. Phys Plasmas 11:3360-3367

35. Yu J, Desaintfuscien M, Plumelle F (1989) Ion density limitation in a Penning trap due to the combined effect of asymmetry and space charge. Appl Phys B 48:51-54

36. Kretzschmar M (1990) A Theory of Anharmonic Perturbations in a Penning trap. Zeitschrift für Naturforschung A 45:965-978

37. Kretzschmar M (1992) Single particle motion in a Penning trap: description in the classical canonical formalism. Phys Scr 46:544-554 